\title{
Patientenberatung via Internet unzulässig
}

Nach einem Urteil des Oberlandesgerichts (OLG) Köln ist eine medizinische Beratung im Internet rechtswidrig (Urteil vom 10. August 2012, Az.: 6 U 235/11). Der Fall: Eine Fachärztin für Gynäkologie beteiligte sich an einem Internetauftritt unter dem $\mathrm{Na}$ men "Gesundheitsberatung.de". Über diese Plattform konnten sich Patienten an Ärzte unterschiedlicher Fachrichtungen unter der Rubrik "Sie fragen - Experten antworten" wenden. Ein Wettbewerbsverein erhob daraufhin Unterlassungsklage wegen des Verstoßes gegen das Heilmittelwerbegesetz (HWG).
In seiner Urteilsbegründung beruft sich das OLG auf einen Verstoß gegen §9 HWG, wonach „eine Werbung für die Erkennung oder Behandlung von Krankheiten, Leiden, Körperschäden oder krankhaften Beschwerden, die nicht auf eigener Wahrnehmung an dem zu behandelnden Menschen ... beruht (Fernbehandlung)" unzulässig ist. Im vorliegenden Fall wurden den Experten von den Patienten medizinische Fragen gestellt, die detailliert und individuell mit einer Therapieempfehlung beantwortet wurden.

\section{MMW Kommentar}

Das Urteil kann in vielfältiger Weise übertragen werden und z. B. auch zur Anwendung kommen, wenn Ärzte derartige Auskünfte im Rahmen von Aktionen geben, wie sie in letzter Zeit häufig von Zeitungen oder Zeitschriften durchgeführt werden. Zulässig sind in solchen Fällen lediglich allgemein gehaltene Gesundheitstipps, aber keine konkrete Beratung. Nach Auffassung der Richter müssen sich solche Auskünfte individuell auf die Empfehlung beschränken, sich erneut untersuchen zu lassen.

\section{Hyperbare $\mathrm{O}_{2}$-Therapie keine Kassenleistung}

- Nach einem Urteil des Sozialgerichts (SG) Marburg müssen Vertragsärzte und Vertragspsychotherapeuten mit dem (teilweisen) Entzug ihrer Zulassung rechnen, wenn sie ihrem Versorgungsauftrag für gesetzlich Krankenversicherte nicht im vollen Umfang nachkommen.

Im konkreten Fall wurde einer Psychotherapeutin vom Zulassungsausschuss der hälftige Versorgungsauftrag entzogen, weil sie über drei Jahre hinweg mit zwei Ausnahmen stets weniger als vier Wochen- stunden pro Quartal Leistungen für GKVVersicherte abgerechnet hatte. Als Begründung gaben die Richter an, dass im Bundesmantelvertrag vorgeschrieben ist, dass ein Vertragsarzt oder -psychotherapeut für Kassenpatienten mindestens 20 Sprechstunden pro Woche in seiner Praxis anbieten muss. Maßgeblich für das tatsächliche Leistungsverhalten seien dabei allein die abgerechneten Leistungen (SG Marburg, Urteil vom 14. November 2012, AZ: S 12 KA 879/11).

\section{MMW Kommentar}

Auch wenn sich das Urteil auf die Tätigkeit einer Psychotherapeutin bezieht, ist es auf den ärztlichen Bereich voll übertragbar. Beachtenswert ist dabei, dass ein solches Entzugsverfahren auf den abgerechneten Leistungen und damit den dort festgelegten Plausibilitätszeiten beruht. So gesehen stellt der relativ hohe Pauschalierungsgrad im hausärztlichen Bereich einen Schutz gegen solche Maßnahmen dar, da basierend auf den Quartalszeiten durch- aus auch bei geringer tatsächlicher Wochenarbeitszeit eine durchschnittlich ausreichend hohe Stundenzahl entstehen kann. Vorsicht ist deshalb aber bei Gemeinschaftspraxen geboten. Werden die Quartalspauschalen in zu hohem Maße einem einzelnen Gemeinschaftspraxispartner (per LANR) zugeordnet, kann es zu einer zumindest rechnerischen Schieflage kommen. Darauf sollte man ggf. argumentativ vorbereitet sein.
Das Sozialgericht Aachen hat entschieden, dass die ambulant durchgeführte hyperbare Sauerstofftherapie (HBO) nicht zum Leistungskatalog der GKV gehört.(Sozialgericht Aachen, Urteil vom 4. September 2012, AZ: S 13 KIR 115/12)

\section{MMW Kommentar}

Das Urteil schafft Klarheit darüber, wer die Kosten für eine solche Behandlung zu tragen hat. Es hat aber auch Auswirkung auf die künftige, von der KBV geplante "Trennung" in typische und atypische Hausärzte. Danach sollen Hausärzte, die nach Auffassung der KBV hausarztuntypische Leistungen, wie z. B. Akupunktur, erbringen, für den betreffenden Fall nur ein reduziertes Honorar aus der Gesamtvergütung erhalten. Individuelle Gesundheitsleistungen (IGeL), zu denen die $H B O$ gezählt werden kann, spielen dabei aber keine Rolle. Da auch die Akupunktur nur bei ganz bestimmten Indikationen eine GKV-Leistung darstellt, sollte man spätestens nach Einführung des neuen EBM genau zwischen GKV- und IGeLeistung trennen. 